\title{
SHAPE FROM SILHOUETTE CONSENSUS AND PHOTO-CONSISTENCY
}

\author{
Gloria Haro \\ Universitat Pompeu Fabra
}

\begin{abstract}
We propose a 3D reconstruction algorithm based on silhouettes and color images. It is robust to inconsistent silhouettes, often common in real applications due to occlusions, errors in the background subtraction, noise or even calibration errors. The recovery of the shape that best fits the available data is formulated as a continuous energy minimization problem. The energy is based on the error between the silhouettes and the shape plus a regularization term based on a photo-consistency measure that places the surface at photoconsistent locations. The visibility is modeled as a function of the shape. The proposed photo-consistency measure takes visibility into account, although the presented variational framework can use different photo-consistency computations.
\end{abstract}

Index Terms - 3D reconstruction, multi-view, shape from silhouette, photo-consistency, visibility.

\section{INTRODUCTION}

The 3D reconstruction from multiple views is one of the main problems in computer vision that has many applications, like human body motion analysis, 3D localization and navigation or augmented reality. Two different kind of approaches have been used to solve the problem: shape from silhouettes (SfS) and shape from multi-view stereo.

SfS methods based on the visual hull [1, 2, 3, 4, 5] usually provide a fast and simple reconstruction but will produce incomplete shapes in situations where the silhouettes are not consistent due to segmentation errors. That is why recently there has been a growing interest in proposing SfS methods robust to inconsistent silhouettes [6, 7, 8, 9, 10, 11, 12, 13]. However, an inherent problem of the SfS techniques is that they can not recover some of the concavities present in the shape.

On the other hand, multi-view stereo methods use the photo-consistency of voxels and are able to recover those concavities. Common photo-consistency measures assume a Lambertian hypothesis and thus those methods fail to reconstruct parts of the surface shape affected by specularities, but also in cases of occlusions and lack of texture [14, 15].

Thanks to the Spanish Ministerio de Economía y Competitividad, under the Ramón y Cajal program and the MTM2012-30772 project, the Generalitat de Catalunya under the GRC 2009 SGR 773 project, and the European Research Council under the ERC grant agreement no319899 for funding.
As shown in previous works $[16,17,18,19,14,20]$ the fusion of consistent silhouettes and stereo information greatly improves the results compared to using any of these types of data separately. The mentioned works impose and exact silhouette consistency assuming that the silhouettes do not have errors. This assumption is no longer valid in many applications where images are taken in a non-controlled environment [15] and possible source of errors arise: occlusions, errors in the background subtraction (due to shadows, illumination changes, similar colors in foreground and background), noise or even calibration errors. As far as we know the only work that combines photo-consistency and non-perfect silhouettes is [21], where the authors consider uncertainties in the silhouettes. On the other hand, [16] just omits the constraints coming from silhouettes with a low confidence.

We propose a $3 \mathrm{D}$ reconstruction method that uses color images and silhouettes. The difference with previous works is that our method is robust to inconsistent silhouettes since we do not impose an exact silhouette match. Instead, we minimize the reprojection error of the shape with respect to the silhouettes while at the same time seeking for a smooth surface located at most photo-consistent locations.

\section{COMBINING PHOTO-CONSISTENCY AND INCONSISTENT SILHOUETTES}

The data in our problem are the calibrated color images and the segmented foreground objects in each of the $N_{c}$ views. Let us denote the color images by $I_{i}: \mathcal{D} \subset \mathbb{R}^{2} \rightarrow \mathbb{R}^{3}$, $i=1, \ldots, N_{c}$. On the other hand, the segmented objects are represented by binary images, $S_{i}: \mathcal{D} \subset \mathbb{R}^{2} \rightarrow\{0,1\}$, $i=1, \ldots, N_{c} . S_{i}$ takes the value one at points which belong to the segmented foreground objects. We will refer to this set of points as the silhouette, and to $S_{i}$ as the $i$-th silhouette image (i.e. the characteristic function of the silhouette).

Consider a volume $\Omega \subset \mathbb{R}^{3}$ and a binary function that defines the space occupancy, $C: \Omega \rightarrow\{0,1\}$, i.e. the characteristic function of the shape in the $3 \mathrm{D}$ space. Our purpose is to recover $C$ from the available data: the color and the silhouette images in the different views while being robust to possible errors and inconsistencies between the silhouettes.

One of the consolidated approaches for solving the 3D reconstruction problem is to use a weighted area functional; as proposed in [22], the reconstructed shape is the one with min- 
imal surface, where the weight of each surface point is given by a photo-consistency measure. In this way, boundaries of the surface at photo-consistent locations are preferred. But the main problem of this formulation is that the trivial solution is the empty set. There have been different proposals to avoid this problem: for example the use of ballooning terms [20], or constraints based on the visual hull [17, 19, 14]. The reconstruction method proposed in [16] is also based on a photo-consistency-weighted area functional where the surface is implicitly represented using the characteristic function of the shape, $C$. Being $\rho(z)$ the photo-consistency measured at point $z \in \Omega$, the shape is found by minimizing the following functional with respect to $C, \int_{\Omega} \rho(z)|\nabla C| d z$, and imposing exact consistency of $C$ with the silhouettes. The latter means that for pixels inside the silhouette (pixels where $S_{i}$ is one) at least one of the $3 \mathrm{D}$ points along the visual ray of the pixel should be occupied by the shape; whereas for pixels outside ( $S_{i}$ is zero), none of the points on the visual ray should be occupied.

Our proposal may be interpreted as a robust version of the work presented in [16] since we consider inconsistent silhouettes. Thus, instead of finding the shape that exactly matches the silhouettes we minimize the reprojection error of the shape with respect to the silhouettes. As in [16], we also find a shape with minimal surface favoring its location at photo-consistent 3D points. The present work is an extension of our previous model [13], where we only consider as data the inconsistent silhouettes but not the color images.

Let us define the error between the shape $C$ at point $z \in \Omega$ and the silhouette image $S_{i}$ of the $i$-th view:

$$
\mathcal{E}_{i}\left(C, S_{i}\right)(z):=(1-C(z)) S_{i}\left(P_{i} z\right)+C(z)\left(1-S_{i}\left(P_{i} z\right)\right),
$$

where $P_{i} z \in \mathcal{D}$ is the projection of point $z \in \Omega$ to camera $i$. The first term expresses the error produced by an empty location $(C(z)=0)$ and the second one the error of an occupied location $(C(z)=1)$. The error $\mathcal{E}_{i}\left(C, S_{i}\right)$ simplifies to:

$$
\mathcal{E}_{i}\left(C, S_{i}\right)(z)=S_{i}\left(P_{i} z\right)+\left(1-2 S_{i}\left(P_{i} z\right)\right) C(z)
$$

In the following, we will denote by empty visual ray a visual ray which does not intersect with the shape, i.e. a visual ray where all the points $z$ in it verify that $C(z)=0$. Let $\tilde{\Omega}_{i}$ be the set of all empty visual rays in $\Omega$, with respect to camera $i$. On the other hand, if the visual ray intersects the shape it is called an occupied visual ray. The idea is then to find the function $C$ that minimizes, for every camera, the error (1) in all the visible points, that is, the points belonging to empty visual rays (i.e. $z \in \tilde{\Omega}_{i}$ ) and those located on the visible surface in the occupied visual rays. Among the different minimizers we are interested in those that have minimum photoconsistency-weighted surface area, thus regularizing the solution while locating the shape surface at photo-consistent locations. Taking this into account we can find the shape $C$ as the one that minimizes the following energy functional:

$$
\begin{array}{r}
\sum_{i=1}^{N_{c}}\left[\int_{\tilde{\Omega}_{i}} \mathcal{E}_{i}\left(C, S_{i}\right) d z+\int_{\Omega \backslash \tilde{\Omega}_{i}} \mathcal{E}_{i}\left(C, S_{i}\right) V_{i} d z\right]+ \\
\alpha \int_{\Omega} \rho\left(z, V_{1}, \ldots, V_{N_{c}}\right)|\nabla C| d z,
\end{array}
$$

where $V_{i}: \Omega \rightarrow[0,1]$ characterizes the visible surface of the shape in camera $i: V_{i}(z) \neq 0$ indicates that point $z$ is visible from camera $i$. Both, the visible surface $V_{i}$ and the set of empty visual rays $\tilde{\Omega}_{i}$ depend on the shape $C$. The mathematical relationship of these functions and sets is detailed in the following subsections. The gradient of $C, \nabla C$, is considered in the distributional sense. The last term of (2) is the weighted total variation (TV) of $C$ : if $C$ is the characteristic function of the shape, then the (weighted) TV computes the (weighted) surface area of the shape. The constant $\alpha$ is the regularization parameter and balances the error terms (fitness to silhouette images) and the surface term (surface regularization and fitness to color images).

Note that the photo-consistency measure we use in (2) depends on the visibility of the points and thus takes occlusion into account. On the other hand, the photo-consistency used in [16] can be precomputed because it does not depend on the visibility; they use the photo-consistency proposed in [19] which includes robustness to occlusions without using explicitly the visibility. Nevertheless, the energy proposed in (2) admits any photo-consistency function, even if it does not depend on the visibility, that is, a function of the type $\rho(z)$.

\subsection{Visibility}

Following [13] and the original idea of [23], we define the depth hull as the function $D_{i}$ which solves the following PDE:

$$
\partial_{r_{i, z}} D_{i}=\max \left(0, H\left(1-D_{i}\right) \partial_{r_{i, z}} C\right)
$$

where $H$ is the Heaviside function, together with the boundary constraint that $D_{i}(z)=0$ in points $z$ located at the boundaries $\partial \Omega$ that first intersect the visual rays when we travel these rays in increasing order of the distances to the camera. Let us denote these boundaries by $\Gamma_{i}$ (depicted in blue in Fig. 1). Equation (3), together with the boundary condition $\left.D_{i}\right|_{\Gamma_{i}}=0$, ensures that $\partial_{r_{i, z}} D_{i} \geq 0$ and that whenever $C$ is a characteristic function so is $D_{i}$, and the non-zero directional derivative of the depth hull, $\partial_{r_{i, z}} D_{i}$, is aligned with the non-zero directional derivative of the shape, $\partial_{r_{i, z}} C$, closer to camera $i$. The visible surface in the energy (2) is then localized by $V_{i}=\left|\partial_{r_{i, z}} D_{i}\right|$.

\subsection{Empty and occupied visual rays}

As shown in the previous subsection, the depth hull $D_{i}$ characterizes the visible surface. Now we will see how it may identify the empty and occupied rays as well. Two different types of volume domain boundaries for $D_{i}$ can be considered: 
the boundaries where the closest points (within $\Omega$ ) to the camera center lie, the set $\Gamma_{i}$, and those others where the furthest points are located. Notice that by definition, the value of $D_{i}$ is always 0 in the closest boundaries, $\Gamma_{i}$, but changes in the furthest boundaries: being 0 if the visual ray is empty and 1 if it is occupied. Then, the functional (2) can be written in the following way:

$$
\begin{aligned}
E\left(C, D_{1}, \ldots, D_{N_{c}}\right)= & \sum_{i=1}^{N_{c}}\left[\int_{\Omega} \mathcal{E}_{i}\left(C, S_{i}\right)\left(1-D_{i}\left(F_{i} z\right)\right) d z\right. \\
& \left.+\int_{\Omega} \mathcal{E}_{i}\left(C, S_{i}\right)\left|\partial_{r_{i, z}} D_{i}\right| d z\right] \\
& +\alpha \int_{\Omega} \rho\left(z, V_{1}, \ldots, V_{N_{c}}\right)|\nabla C| d z
\end{aligned}
$$

where $F_{i} z$ is the projection of $z$ to the furthest point in $\Omega$ from the optical center of camera $i$ that belongs to the same visual ray as $z$ (see Fig. 1). If $D_{i}\left(F_{i} z\right)=0$, then the visual ray is empty, whereas $D_{i}\left(F_{i} z\right)=1$ indicates an occupied visual ray.

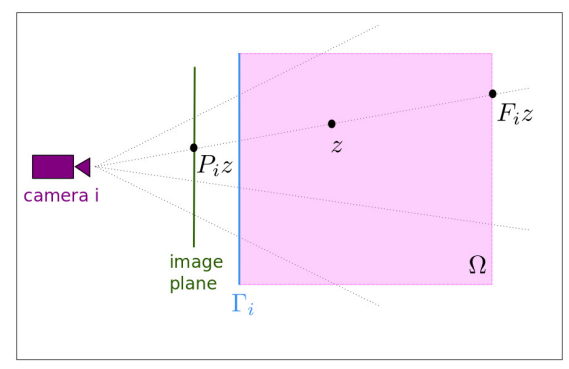

Fig. 1: Example of a 3D domain $\Omega$ and a camera $i$. The boundary $\Gamma_{i}$ (in blue) is the set of points in the boundary of $\Omega$ that first intersect the visual rays when we travel these rays in increasing order of the distances to the camera. Point $z$ projects to $P_{i} z$ in the image plane. Point $F_{i} z$ is the projection of $z$ to the furthest point in $\Omega$ from the optical center of camera $i$ that belongs to the same visual ray as $z$.

\subsection{Relaxed variational problem}

Taking into account both previous characterizations, we propose to solve the following variational problem:

$$
\begin{array}{ll}
\min _{C, D_{i}} & E\left(C, D_{1}, \ldots, D_{N_{c}}\right) \\
\text { s. t. } & C: \Omega \rightarrow\{0,1\} \\
& \left.D_{i}\right|_{\Gamma_{i}}=0, \partial_{r_{i, z}} D_{i}=\max \left(0, H\left(1-D_{i}\right) \partial_{r_{i, z}} C\right) \\
& \forall i=1, \ldots, N_{c} .
\end{array}
$$

Although the functional in (5) is convex in $C$ for fixed $D_{i}$ the variational problem is not convex, since the space of binary functions to which $C$ belongs is non-convex. Therefore we relax the problem by considering that $C \in[0,1]$. We will solve the relaxed variational problem numerically and obtain the solution to the original problem (5) by thresholding the solution of the relaxed problem, as in [16]. Before defining the relaxed problem let us remark that the function $D_{i}$ in (3) is thought for a binary $C$. By relaxing the range of possible values for $C$ we could end up with different visible surface points $z$, i.e. $\partial_{r_{i, z}} D_{i}(z) \neq 0$, in the same line of sight. This situation is avoided by using a thresholded version of $C$ with a certain threshold, $C_{t h}$, in (3) (in practice we use $t h=0.95$ ). The relaxed variational problem is then,

$$
\begin{array}{ll}
\min _{C, D_{i}} & E\left(C, D_{1}, \ldots, D_{N_{c}}\right) \\
\text { s. t. } & C: \Omega \rightarrow[0,1] \\
& \left.D_{i}\right|_{\Gamma_{i}}=0, \partial_{r_{i, z}} D_{i}=\max \left(0, H\left(1-D_{i}\right) \partial_{r_{i, z}} C_{t h}\right) \\
& \forall i=1, \ldots, N_{c} .
\end{array}
$$

Similarly as in [16], it can be proved [13] that once we have the solution $C_{r}$ to the relaxed problem (6), for a fixed $D_{i}$, its thresholded version, $C_{t h}$, lies within an energetic bound of the optimal solution, $C_{o p t}$, to the original problem (5).

\section{NUMERICAL METHOD}

The Euler-Lagrange equation associated to the first variation of the energy $E$ with respect to function $C$ is a necessary condition for the minimum of the energy,

$$
\begin{array}{r}
0=\sum_{i=1}^{N_{c}}\left(1-2 S_{i}\left(P_{i} z\right)\right)\left[\left(1-D_{i}\left(F_{i} z\right)\right)+\left|\partial_{r_{i, z}} D_{i}\right|\right] \\
-\alpha \operatorname{div}\left(\rho\left(z, V_{1}, \ldots, V_{N_{c}}\right) \frac{\nabla C}{|\nabla C|}\right) .
\end{array}
$$

We find $C$ solving equation (7) with a successive overrelaxation algorithm as in [16]. The constraints in $C$ may be imposed at each step: values of $C$ outside the interval $[0,1]$ are clipped. For finding $D_{i}$ we apply the constraint on it which is a PDE (3) that can be solved with a one-pass algorithm, with the Depth hull algorithm proposed in [13] (and based on [23]). As in [13], $S_{i}\left(P_{i} z\right)$ is the average of the silhouette image $i$ in the set of projected pixels for voxel $z$, and we use an upwind scheme for the directional derivative. Finally, the resolution of the system (7) and the Depth hull algorithm are iterated until convergence. This is a simple algorithm which convergences in a few iterations. However, this procedure only minimizes the energy with respect to $C$. Part of the future work is to propose a numerical method that minimizes the energy with respect to the shape and the depth hulls.

\subsection{Photo-consistency measure}

We define the photo-consistency measure as:

$$
\rho\left(z, V_{1}, \ldots, V_{N_{c}}\right)=\frac{1}{N_{i j}} \sum_{i=1}^{N_{c}} \sum_{j>i} \rho_{i j}(z) V_{i}(z) V_{j}(z),
$$




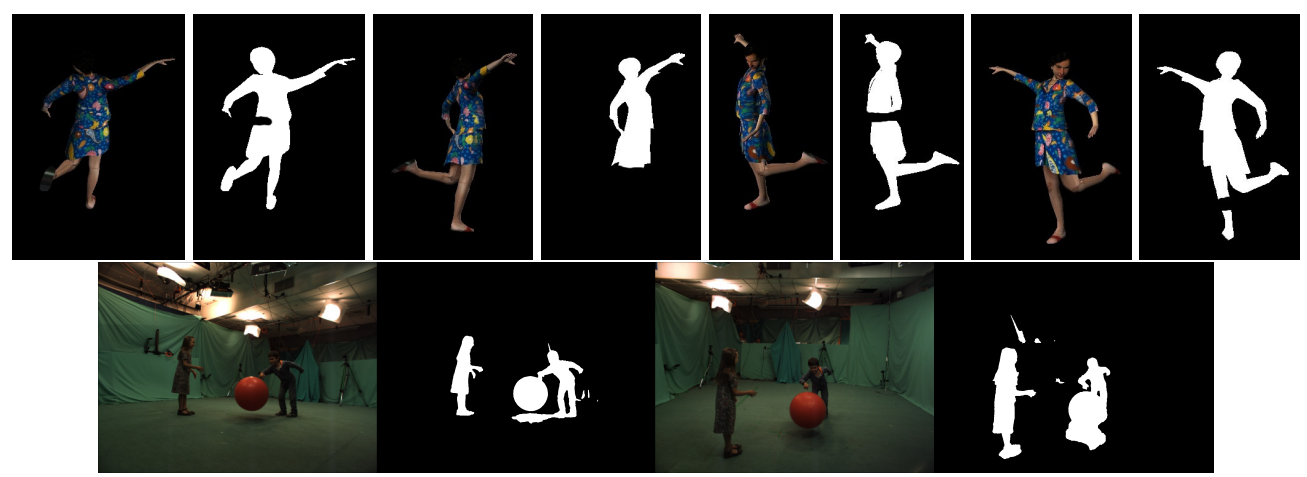

Fig. 2: Top: Synthetic experiment, four of the color images and their corresponding (inconsistent) silhouette images. The rest of the four views do have consistent silhouettes. Bottom: Real experiment, two views and their corresponding silhouette images.

where $N_{i j}=\sum_{i=1}^{N_{c}} \sum_{j>i} V_{i}(z) V_{j}(z)$ and $\rho_{i j}(z)$ is the norm of the difference of the mean projected RGB vectors at camera $i$ and $j$ (normalized by $3 \cdot 128$ ). We use $V_{i}=\left|\partial_{r_{i, z}} D_{i}\right|$ as defined in 2.1. However, if $N_{i j}$ happens to be zero for a specific voxel we have experimentally tested that it is useful to use the same expression (8) with $V_{i} V_{j}=1$ if the angle formed by the visual rays of cameras $i$ and $j$ is less than $45^{\circ}$ and $V_{i} V_{j}=0$ otherwise. If again $N_{i j}=0$, then we set $\rho$ to 1 .

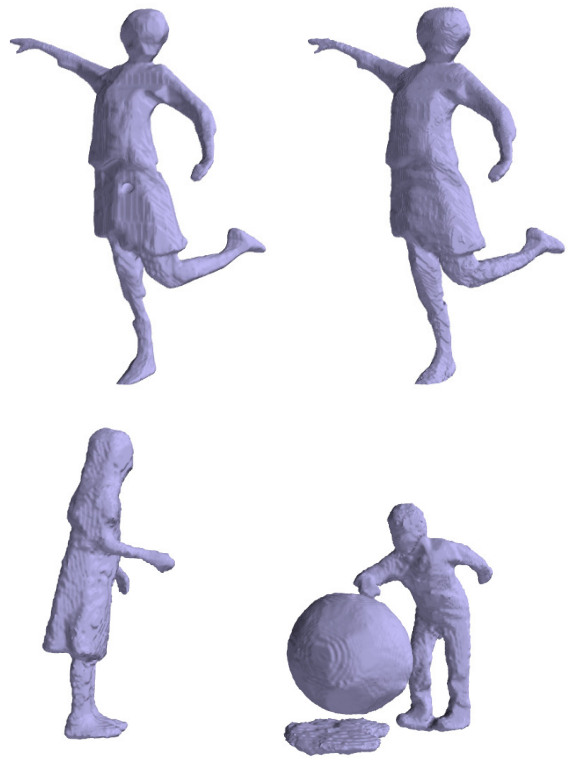

Fig. 3: Recovered shapes, using just silhouettes (top-left) and combining color images and silhouettes (top-right and bottom).

\section{EXPERIMENTS}

The first experiment corresponds to a synthetic data with eight different views, four of the silhouette images have been cor- rupted so as to produce inconsistencies between them (see Fig. 2 top). The 3D reconstruction using the proposed method is shown in Fig. 3, top-right. As it can be seen, the inconsistent parts are better recovered compared to the reconstruction just using the silhouettes [13] (Fig. 3 top-left): the hole in the waist disappears and the indentation in the leg is smoothed. In both cases we have used a volume of $230 \times 226 \times 332$ voxels.

The second experiment uses real data of sixteen views of a two children taken in a studio. ${ }^{1}$ The provided silhouettes are inconsistent due to segmentation errors (Fig. 2 bottom). The recovered shape, Fig. 3 bottom $(275 \times 275 \times 200$ voxels $)$, is robust to silhouette errors.

\section{CONCLUSIONS}

In this work we have proposed a way to combine the color images and the (possible) inconsistent silhouettes so as to find a compatible 3D reconstruction. The novelty with respect to previous methods in the literature is the robustness to inconsistent (or incomplete) silhouettes which are quite common in real applications. Previous works suppose consistent silhouettes and usually impose a perfect match of the shape with the silhouettes. In this case we minimize the reprojection error of the shape with respect to the silhouettes while at the same time seeking for a smooth surface located at most photoconsistent locations.

As a proof of concept we have used a photo-consistency measure that takes visibility into account. However, the variational formulation admits different definitions of the photoconsistency measure. It is part of our future work to test if there are more convenient photo-consistency measures that could help to improve the results.

Another aspect that needs further analysis is the numerical method, as stated in Section 3. Finally we plan to implement the numerical algorithm in CUDA to speed up computations.

\footnotetext{
${ }^{1}$ Data available from http://4drepository.inrialpes.fr/public/datasets
} 


\section{REFERENCES}

[1] B. G. Baumgart, Geometric modeling for computer vision., Ph.D. thesis, CS Departmnet, Stanford University, 1974.

[2] A. Laurentini, "The visual hull concept for silhouettebased image understanding," IEEE Trans. Pattern Analysis and Machine Intelligence, vol. 16, no. 2, pp. 150162, 1994.

[3] K. M. Cheung, T. Kanade, J.-Y. Bouguet, and M. Holler, "A real time system for robust 3d voxel reconstruction of human motions," in Proc. of the IEEE Conference on Computer Vision and Pattern Recognition, 2000, vol. 2, pp. $714-720$.

[4] J. Franco and E. Boyer, "Efficient polyhedral modeling from silhouettes," IEEE Trans. on Pattern Analysis and Machine Intelligence, vol. 31, no. 3, pp. 414-427, 2009.

[5] W. Matusik, C. Buehler, and L. Mcmillan, "Polyhedral visual hulls for real-time rendering," in Proc. of Twelfth Eurographics Workshop on Rendering, 2001, pp. 115125.

[6] D. Snow, P. Viola, and R. Zabih, "Exact voxel occupancy with graph cuts," in Proc. of IEEE Conference on Computer Vision and Pattern Recognition, 2000, vol. 1, pp. 345-352.

[7] N. Campbell, G. Vogiatzis, C. Hernández, and R. Cipolla, "Automatic 3d object segmentation in multiple views using volumetric graph-cuts," in Proc. of British Machine Vision Conference, 2007, pp. 530-539.

[8] W. Lee, W. Woo, and E. Boyer, "Identifying foreground from multiple images," in Proc. of Asian Conference on Computer Vision, 2007, pp. 580-589.

[9] J. Franco and E. Boyer, "Fusion of multi-view silhouette cues using a space occupancy grid," in Proc. of IEEE International Conference on Computer Vision, 2005, pp. 1747-1753.

[10] J. L. Landabaso, M. Pardàs, and J. R. Casas, "Shape from inconsistent silhouette," Computer Vision and Image Understanding, vol. 112, no. 2, pp. 210-224, 2008.

[11] L. Díaz-Más, R. Muñoz-Salinas, F.J. Madrid-Cuevas, and R. Medina-Carnicer, "Shape from silhouette using dempster-shafer theory," Pattern Recognition, vol. 43, pp. 2119-2131, 2010.

[12] G. Haro and M. Pardàs, "Shape from incomplete silhouettes based on the reprojection error," Image and Vision Computing, vol. 28, no. 9, pp. 1354-1368, 2010.
[13] G. Haro, "Shape from silhouette consensus," Pattern Recognition, vol. 45, no. 9, pp. 3231-3244, 2012.

[14] S. Sinha and M. Pollefeys, "Multi-view reconstruction using photo-consistency and exact silhouette constraints: A maximum-flow formulation," in Proc. IEEE International Conference on Computer Vision, 2005.

[15] L. Guan, J.S. Franco, and M. Pollefeys, "Multi-view occlusion reasoning for probabilistic silhouette-based dynamic scene reconstruction," International journal of computer vision, vol. 90, no. 3, pp. 283-303, 2010.

[16] D. Cremers and K. Kolev, "Multiview stereo and silhouette consistency via convex functionals over convex domains," IEEE Trans. Pattern Analysis and Machine Intelligence, vol. 33, pp. 1161-1174, 2010.

[17] Y. Furukawa and J. Ponce, "Carved visual hulls for image-based modeling," in Proc. of European Conference on Computer Vision, pp. 564-577. 2006.

[18] J Isidro and Stan Sclaroff, "Stochastic refinement of the visual hull to satisfy photometric and silhouette consistency constraints," in Proc. of IEEE International Conference on Computer Vision, 2003, pp. 1335-1342.

[19] C. Hernández and F. Schmitt, "Silhouette and stereo fusion for 3D object modeling," Computer Vision and Image Understanding, vol. 96, no. 3, pp. 367-392, 2004.

[20] G. Vogiatzis, P.H.S Torr, and R. Cipolla, "Multi-view stereo via volumetric graph-cuts," in Proc. IEEE Conference on Computer Vision and Pattern Recognition, 2005.

[21] S. Tran and L. Davis, "3d surface reconstruction using graph cuts with surface constraints," in Proc. of European Conference on Computer Vision, pp. 219-231. 2006.

[22] O. Faugeras and R. Keriven, "Complete dense stereovision using level set methods," in Proc. of European Conference on Computer Vision, 1998, pp. 379-393.

[23] Y. H. R. Tsai, L. T. Cheng, S. Osher, P. Burchard, and G. Sapiro, "Visibility and its dynamics in a pde based implicit framework," Journal of Computational Physics, vol. 199, no. 1, pp. $260-290,2004$. 